\title{
Cosmic ray fluxes and the role of sub-dominant source populations to the positron excess
}

\author{
Jagdish C. Joshi* \\ University of Johannesburg \\ E-mail: jjagdish@uj.ac.za \\ Soebur Razzaque \\ University of Johannesburg \\ E-mail: srazzaque@uj.ac.za
}

\begin{abstract}
Interactions of cosmic-ray nuclei is the most well-understood contribution to the observed positron flux on Earth. Various scenarios have been proposed, including sources in the spiral arms of the galaxies and nearby isolated sources, which can contribute to the measured flux. In our work we focus on sources such as the gamma-ray novae which can contribute to the observed positron flux, although sub-dominant to the supernova remnants or pulsar-wind nebulae overall, but crucial in the 10 s of $\mathrm{GeV}$ energy range.
\end{abstract}

The 35th International Cosmic Ray Conference,

12-20 July, 2017

BEXCO, BUSAN, KOREA

\footnotetext{
* Speaker.
} 


\section{Introduction}

Our Galaxy is a continuous source of most of the cosmic-ray charged particles observed on Earth. These cosmic rays, which can be classified into primary and secondary particles as well as the matter particles (proton, Helium, electron, etc.) and antimatter particles (antiproton, positron, etc.), convey a wealth of information about their sources in the Galaxy and intervening medium. Recent data from the balloon-borne detector High Energy Antimatter Telescope (HEAT) [1], satellite-borne detectors Payload for Antimatter Matter Exploration and Light-nuclei Astrophysics (PAMELA) [2], Fermi-Large Area Telescope (LAT) [3] as well as the Alpha Magnetic Spectrometer (AMS) [4] onboard the International Space Station has led to significant development in understanding the properties of the cosmic ray sources and interstellar medium (ISM). Observations of cosmic-ray positron flux by HEAT [5], PAMELA [6], AMS [4] and Fermi-LAT [7] have shown an excess in the $\approx 10-500 \mathrm{GeV}$ energy range, different than expected from propagation and interactions of cosmic-ray protons in the ISM. This excess of positron fraction in the data has been used to understand its origin via dark matter particles [8-11] and via astrophysical sources [12-21]. An astrophysical interpretation is widely favored over dark matter annihilation models at present, although it requires additional mechanisms and/or sources of positrons.

\section{Model setup}

In our model we considered a diffusive reacceleration with no convection scenario for propagation of cosmic rays using the three dimensional model in the DRAGON code. We did-not consider convection process during the cosmic-ray transport in our Galaxy, as this process lowers the $\mathrm{B} / \mathrm{C}$ ratio in the $1-10 \mathrm{GeV}$ energy range which needs to be recovered by using large values of Alfven speed. The magnetic field structure has been selected in the DRAGON code of type Pshirkov, in which the disk component has a value $2 \mu \mathrm{G}$ and the halo component can be in a range $2-5 \mu \mathrm{G}$ based on the radio synchrotron emission of the Galaxy [22]. We have used the Galactic magnetic field disk component value as $2 \mu \mathrm{G}$, halo component value as $4 \mu \mathrm{G}$ and the turbulent component value as $10 \mu \mathrm{G}$, respectively. We have taken the exponential mode of diffusion coefficient in the DRAGON code which varies with particle rigidity $\rho$ and the Galaxy vertical height $z$ [23].

In this propagation scenario we calculate the secondary nuclei, namely the Boron to Carbon ratio $[24,25]$ to fix the diffusion coefficient and the size of the Galactic halo [26]. In a recent AMS result it has been shown that above rigidity $\rho=65 \mathrm{GV}, \delta$ takes a value $0.333 \pm 0.014$ (stat) \pm 0.005 (syst) [25]. Below $10 \mathrm{GeV}$, the effect of solar modulation on the charged particles can be adopted to fit the observational data [27].

\section{Fluxes of nuclei and cosmic-ray powers}

We have plotted the Boron to Carbon flux ratios in the left panel of Fig. 1, while in the right panel we have plotted the proton flux along with observed data. The proton flux detected by AMS02 and PAMELA data can be explaind by different activity of the solar medium ${ }^{1}$. The solid (dotted) lines are fluxes after (before) taking into account solar modulation. Our choice of model parameters

\footnotetext{
${ }^{1}$ All data are taken from the database [28] unless otherwise specified.
} 


\begin{tabular}{|c|c|c|c|c|c|c|c|}
\hline Parameters & $\delta$ & $D_{0}\left(\mathrm{~cm}^{2} / \mathrm{s}\right)$ & $v_{A}(\mathrm{~km} / \mathrm{s})$ & $\eta$ & $\gamma(p)$ & $\gamma\left(e^{-}\right)$ & $\gamma\left(e^{ \pm}\right)$ \\
\hline Value & 0.45 & $2.8 \times 10^{28}$ & 19 & -0.005 & $2.0 / 2.32$ & $1.91 / 2.71 / 2.36$ & $1.85 / 2.32$ \\
\hline
\end{tabular}

Table 1: Galaxy parameters for a halo of size $\mathrm{x}, \mathrm{y}, \mathrm{z}(=12 \mathrm{kpc}, 12 \mathrm{kpc}, 8 \mathrm{kpc})$, used in the solution for the transport equation of cosmic rays. All the nuclei follow the same spectrum with a rigidity break at $7 \mathrm{GV}$, the primary electron has two breaks at $6.2 \mathrm{GV}$ and $85 \mathrm{GV}$ and the additional population has a break at $3.8 \mathrm{GV}$.

fits the $\mathrm{B} / \mathrm{C}$ ratio data from both the AMS-02 [25] and PAMELA [24] quite well in the whole observed energy range. This requires the index of the diffusion coefficient to be $\delta=0.45$, close to the Kraichnan turbulence spectrum rather than the Kolmogorov turbulence spectrum of $\approx 0.33$ reported in [25].

We calculate the total powers in cosmic-ray components, using the spectra in Table 1, by injecting and propagating these particles using the DRAGON code for $T=64$ Million years in a volume $V=1.15 \times 10^{3} \mathrm{kpc}^{3}$ of the Galaxy, as

$$
P=\frac{V}{T} \frac{4 \pi}{c} \int_{E_{1}}^{E_{2}} n(E) E d E .
$$

Here $E_{1}$ and $E_{2}$ are the two limiting energies in the spectrum. In our calculations, the approximate total power required in the protons is $10^{56} \mathrm{GeV} / \mathrm{Myr}$, in primary electrons is $2.8 \times 10^{54} \mathrm{GeV} / \mathrm{Myr}$ and in the additional $e^{ \pm}$populations is $1.5 \times 10^{53} \mathrm{GeV} / \mathrm{Myr}$.
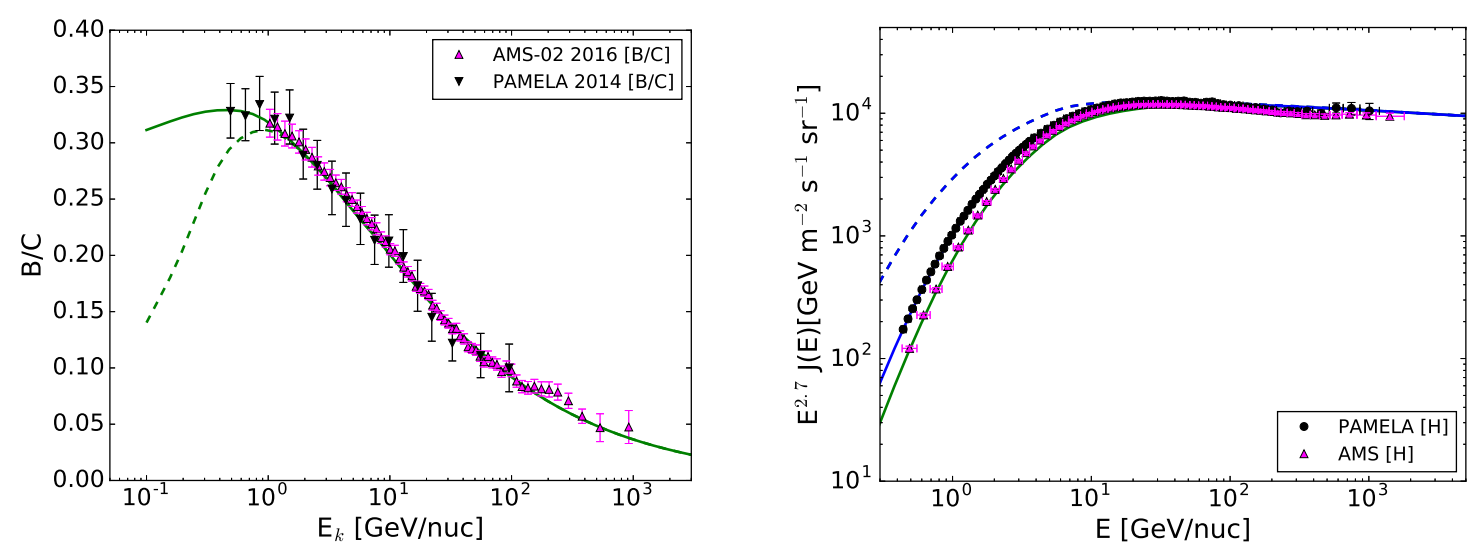

Figure 1: Left panel: B/C ratio calculation using the DRAGON code and plotted against the AMS [25] and PAMELA [24] data (From Joshi et al.,2017 [29]). Right panel: The proton flux plotted against the PAMELA [30] and AMS data [31]. The PAMELA proton data can be explained with a lower solar modulation $\phi=0.42 \mathrm{GV}$ (blue solid line), while the AMS-02 data requires a stronger solar modulation $\phi=0.65$ GV (green solid line). The solid (dashed) lines are fluxes with (without) solar modulation taken into account.

\section{Contributions from nearby sub-kpc pulsars}

We have selected nearby pulsars from the Australia Telescope National Facility (ATNF) cata$\operatorname{logue}^{2}$ with parameters taken from there as well as from [32,33] and we have listed those in Table 2.

\footnotetext{
${ }^{2}$ http://www.atnf.csiro.au/people/pulsar/psrcat/
} 


\begin{tabular}{|l|l|l|l|l|}
\hline Name & Distance (kpc) & Age $(\mathrm{yr})$ & $\dot{E}(\mathrm{erg} / \mathrm{sec})$ & $E(t)(\mathrm{erg})$ \\
\hline J0633+1746 (Geminga) & $0.25_{-0.08}^{+0.23}$ & $3.42 \times 10^{5}$ & $3.2 \times 10^{34}$ & $4.4 \times 10^{48}$ \\
B1055-52 & $0.35_{-0.15}^{+0.15}$ & $5.35 \times 10^{5}$ & $3 . \times 10^{34}$ & $1.0 \times 10^{49}$ \\
\hline
\end{tabular}

Table 2: Pulsar parameters based on the ATNF catalogue. Geminga and B1055-52 dominantly contribute to the electron-positron fluxes in the $100 \mathrm{GeV}-1 \mathrm{TeV}$ range in our model.

If $\eta_{e^{ \pm}}$is the efficiency for conversion of spin-down energy of a pulsar into electron-positron pairs, then we can estimate emitted energy in $e^{ \pm}$fluxes as [13],

$$
E(t) \simeq \eta_{e^{ \pm}} \dot{E} \frac{t}{\tau_{0}}
$$

Here $\tau_{0} \sim 10^{4}$ is the luminosity decay time for mature pulsars and $\dot{E}$ is the spin-down luminosity for a given pulsar. We have taken $\eta_{e^{ \pm}}=0.4$ in Table 2 .

\section{Electron-positron flux levels and positron excess}
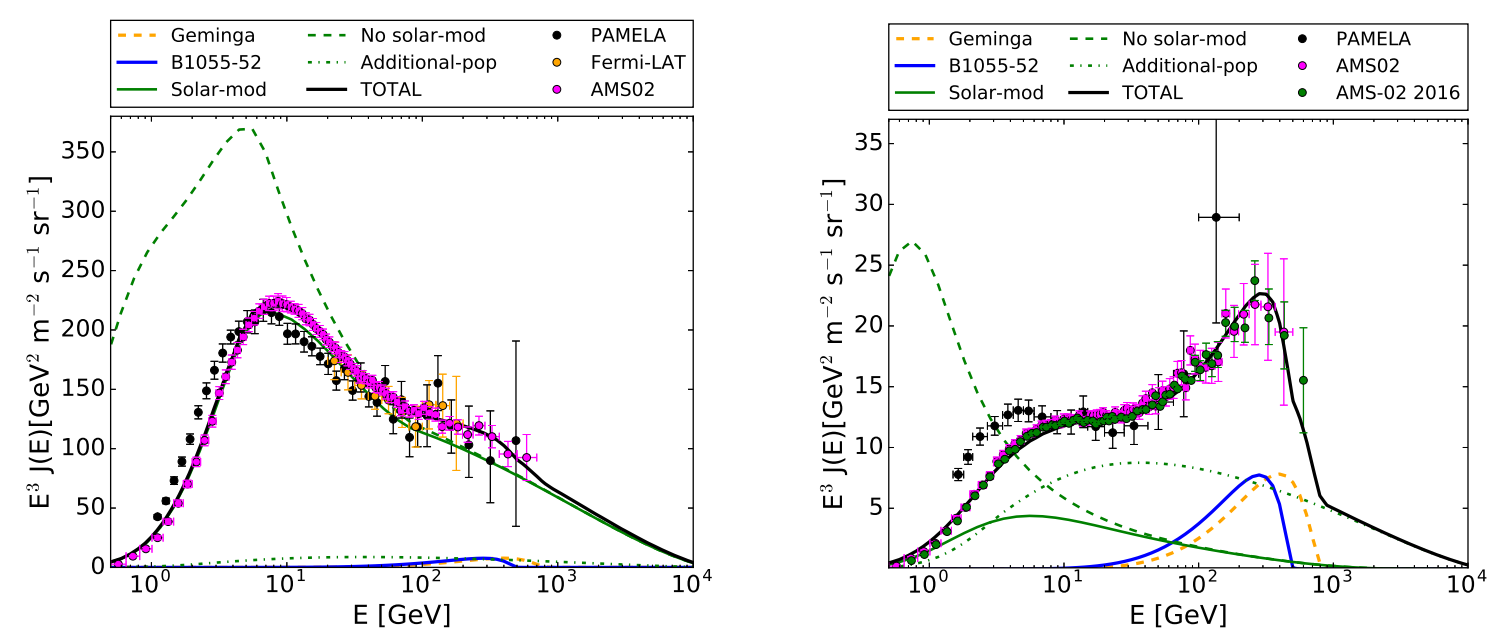

Figure 2: Left panel: Electron flux data from PAMELA [34], Fermi-LAT [7] and AMS-02 [35] (From Joshi et al.,2017 [29]). Right panel: Positron Flux data from PAMELA [6] and AMS-02 [35]). Also shown are our model fluxes with black solid line for the total flux in both panels. See main text for more details.

In Figure 2 the electron and positron flux coming from Galaxy due to cosmic ray interactions with the ISM, primary electron source contribution, primary $e^{ \pm}$pair emitters (additional population) and nearby source contribution have shown. The total addititon of all processes explains the AMS observations. To fit the positron flux at high energies we require a diffusion coefficient 28 times smaller for the diffusion of electrons and positrons from nearby sources than used for the Galactic diffusion of cosmic rays in the DRAGON code. We have assumed that Geminga and B1055-52 are contributing 40\% $\left(\eta_{e^{ \pm}}=0.4\right)$ of their spin-down power into electron-positron pair emission, as mentioned earlier. We have also taken an injection spectral index of 1.89 for both of these sources with an exponential folding energy of $1.3 \mathrm{TeV}$ for Geminga and $1.5 \mathrm{TeV}$ for B105552, which fit the observational data. Please see the earlier work [29] for a full description. 


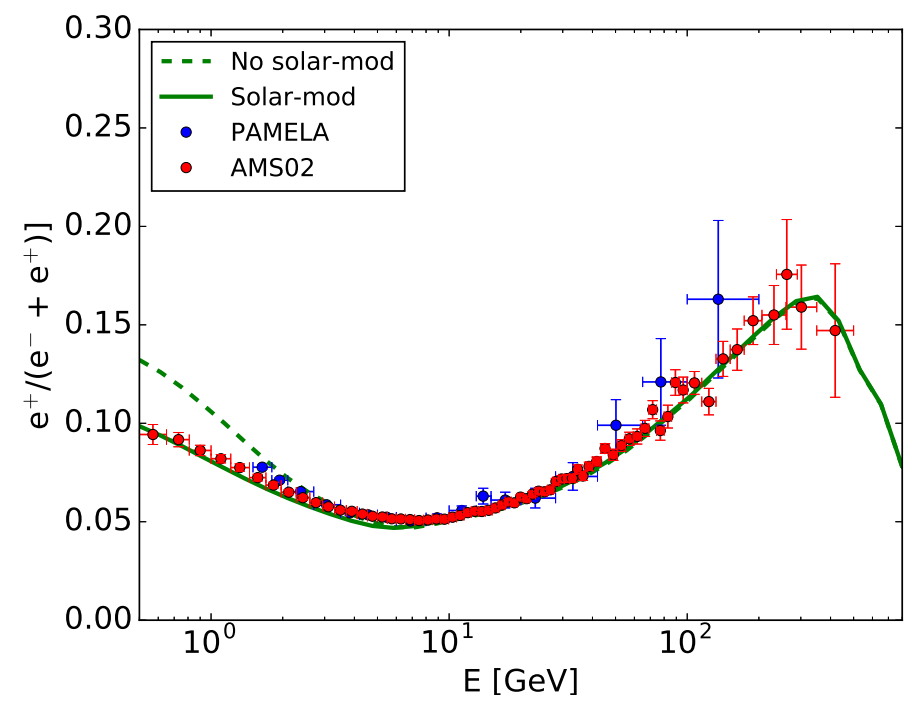

Figure 3: Positron fraction based on our model (solid line) plotted against data from PAMELA [6] and AMS-02 [36].

In Fig. 3 we have plotted the fractional positron flux of the total electron and positron flux. The solid (dashed) line corresponds to the flux model with (without) solar modulation taken into account. Our model fits both the AMS-02 data [35] and PAMELA data [6] rather well.

\section{Summary and Discussion}

The Proton flux we have calculated using the diffusion-reacceleration model in the DRAGON code can fit the AMS-02 data and PAMELA data with higher $(\phi=0.65 \mathrm{GV})$ and lower $(\phi=$ $0.42 \mathrm{GV}$ ) solar modulation activity respectively. We found that the observed spectrum of electrons required two breaks for its successfull interpretation. The low energy break is due to electrons cooling in the Galactic magnetic field, while the second break, after which the flux is harder, might have an origin in the source population of cosmic rays or in the energy dependent escape of electrons from their sources. We needed an additional population of sources, distributed similar to the conventional source population but producing primary electron-positron fluxes. A requirement for an additional source population to fit data is not new. Sources such as white dwarf pulsars [37] with magnetic field $\gtrsim 10^{9} \mathrm{G}$ (see, e.g. [38]) can accelerate $e^{ \pm}$to $10 \mathrm{TeV}$ [39]. Other sources such as gamma-ray novae [40] can also possibly accelerate particles to very-high energies [41, 42].

We have also used contributions to the $e^{-}$and $e^{+}$fluxes from the nearby pulsars, as is usually considered, e.g., $[43,44,21]$ to explain observations in the $\gtrsim 100 \mathrm{GeV}-1 \mathrm{TeV}$ range.

\section{References}

[1] S. W. Barwick, J. J. Beatty, C. R. Bower, C. Chaput, S. Coutu, G. de Nolfo et al., Cosmic Ray Positrons at High Energies: A New Measurement, Physical Review Letters 75 (July, 1995) 390-393. 
[2] P. Picozza, A. M. Galper, G. Castellini, O. Adriani, F. Altamura, M. Ambriola et al., PAMELA A payload for antimatter matter exploration and light-nuclei astrophysics, Astroparticle Physics 27 (Apr., 2007) 296-315, [astro-ph/0608697].

[3] W. B. Atwood, A. A. Abdo, M. Ackermann, W. Althouse, B. Anderson, M. Axelsson et al., The Large Area Telescope on the Fermi Gamma-Ray Space Telescope Mission, ApJ 697 (June, 2009) 1071-1102, [0902.1089].

[4] M. Aguilar, G. Alberti, B. Alpat, A. Alvino, G. Ambrosi, K. Andeen et al., First Result from the Alpha Magnetic Spectrometer on the International Space Station: Precision Measurement of the Positron Fraction in Primary Cosmic Rays of 0.5-350 GeV, Physical Review Letters 110 (Apr., 2013) 141102.

[5] S. W. Barwick, J. J. Beatty, A. Bhattacharyya, C. R. Bower, C. J. Chaput, S. Coutu et al., Measurements of the Cosmic-Ray Positron Fraction from 1 to $50 \mathrm{GeV}$, ApJL 482 (June, 1997) L191-L194, [astro-ph/9703192].

[6] O. Adriani, G. C. Barbarino, G. A. Bazilevskaya, R. Bellotti, A. Bianco, M. Boezio et al., Cosmic-Ray Positron Energy Spectrum Measured by PAMELA, Physical Review Letters 111 (Aug., 2013) 081102, [1308.0133].

[7] M. Ackermann, M. Ajello, A. Allafort, W. B. Atwood, L. Baldini, G. Barbiellini et al., Measurement of Separate Cosmic-Ray Electron and Positron Spectra with the Fermi Large Area Telescope, Physical Review Letters 108 (Jan., 2012) 011103, [1109.0521].

[8] I. Cholis, L. Goodenough, D. Hooper, M. Simet and N. Weiner, High energy positrons from annihilating dark matter, PRD 80 (Dec., 2009) 123511, [0809.1683].

[9] L. Bergström, T. Bringmann and J. Edsjö, New positron spectral features from supersymmetric dark matter: A way to explain the PAMELA data?, PRD 78 (Nov., 2008) 103520, [0 808 . 3725].

[10] T. A. Porter, R. P. Johnson and P. W. Graham, Dark Matter Searches with Astroparticle Data, ARAA 49 (Sept., 2011) 155-194, [1104.2836].

[11] I. Cholis and D. Hooper, Dark matter and pulsar origins of the rising cosmic ray positron fraction in light of new data from the AMS, PRD 88 (July, 2013) 023013, [1304.1840].

[12] D. Hooper, P. Blasi and P. Dario Serpico, Pulsars as the sources of high energy cosmic ray positrons, JCAP 1 (Jan., 2009) 025, [0810.1527].

[13] S. Profumo, Dissecting cosmic-ray electron-positron data with Occam's razor: the role of known pulsars, Central European Journal of Physics 10 (Feb., 2012) 1-31, [0812 . 4457].

[14] H. Yüksel, M. D. Kistler and T. Stanev, TeV Gamma Rays from Geminga and the Origin of the GeV Positron Excess, Physical Review Letters 103 (July, 2009) 051101, [0810 . 2784].

[15] C. Venter, A. Kopp, A. K. Harding, P. L. Gonthier and I. Büsching, Cosmic-ray Positrons from Millisecond Pulsars, ApJ 807 (July, 2015) 130, [1506.01211].

[16] J. Feng and H.-H. Zhang, Pulsar interpretation of lepton spectra measured by AMS-02, European Physical Journal C 76 (May, 2016) 229, [1504.03312].

[17] Y.-Z. Fan, B. Zhang and J. Chang, Electron/positron Excesses in the Cosmic Ray Spectrum and Possible Interpretations, International Journal of Modern Physics D 19 (Nov., 2010) 2011-2058, [1008.4646].

[18] S.-J. Lin, Q. Yuan and X.-J. Bi, Quantitative study of the AMS-02 electron/positron spectra: Implications for pulsars and dark matter properties, PRD 91 (Mar., 2015) 063508, [1409.6248]. 
[19] N. J. Shaviv, E. Nakar and T. Piran, Inhomogeneity in Cosmic Ray Sources as the Origin of the Electron Spectrum and the PAMELA Anomaly, Physical Review Letters 103 (Sept., 2009) 111302, [0902.0376].

[20] M. Kachelrieß, S. Ostapchenko and R. Tomàs, Antimatter Production in Supernova Remnants, ApJ 733 (June, 2011) 119, [1103.5765].

[21] D. Hooper, I. Cholis, T. Linden and K. Fang, HAWC Observations Strongly Favor Pulsar Interpretations of the Cosmic-Ray Positron Excess, ArXiv e-prints (Feb., 2017), [1702.08436].

[22] M. S. Pshirkov, P. G. Tinyakov, P. P. Kronberg and K. J. Newton-McGee, Deriving the Global Structure of the Galactic Magnetic Field from Faraday Rotation Measures of Extragalactic Sources, ApJ 738 (Sept., 2011) 192, [1103.0814].

[23] C. Evoli, D. Gaggero, A. Vittino, G. Di Bernardo, M. Di Mauro, A. Ligorini et al., Cosmic-ray propagation with DRAGON2: I. numerical solver and astrophysical ingredients, JCAP 2 (Feb., 2017) 015, [1607.07886].

[24] O. Adriani, G. C. Barbarino, G. A. Bazilevskaya, R. Bellotti, M. Boezio, E. A. Bogomolov et al., Measurement of Boron and Carbon Fluxes in Cosmic Rays with the PAMELA Experiment, ApJ 791 (Aug., 2014) 93, [1407.1657].

[25] M. Aguilar, Precision measurement of the boron to carbon flux ratio in cosmic rays from $1.9 \mathrm{gv}$ to 2.6 tv with the alpha magnetic spectrometer on the international space station, Phys. Rev. Lett. 117 (Nov, 2016) 231102.

[26] A. W. Strong and I. V. Moskalenko, Propagation of Cosmic-Ray Nucleons in the Galaxy, ApJ 509 (Dec., 1998) 212-228, [astro-ph/9807150].

[27] L. J. Gleeson and W. I. Axford, Solar Modulation of Galactic Cosmic Rays, ApJ 154 (Dec., 1968) 1011.

[28] D. Maurin, F. Melot and R. Taillet, A database of charged cosmic rays, A\&A 569 (Sept., 2014) A32, [1302.5525].

[29] J. C. Joshi and S. Razzaque, A self-consistent model of cosmic-ray fluxes and positron excess: Roles of nearby pulsars and a sub-dominant source population, ArXiv e-prints (June, 2017), [1706.01981].

[30] O. Adriani, G. C. Barbarino, G. A. Bazilevskaya, R. Bellotti, M. Boezio, E. A. Bogomolov et al., PAMELA Measurements of Cosmic-Ray Proton and Helium Spectra, Science 332 (Apr., 2011) 69, [1103.4055].

[31] M. Aguilar, D. Aisa, B. Alpat, A. Alvino, G. Ambrosi, K. Andeen et al., Precision Measurement of the Proton Flux in Primary Cosmic Rays from Rigidity 1 GV to 1.8 TV with the Alpha Magnetic Spectrometer on the International Space Station, Physical Review Letters 114 (May, 2015) 171103.

[32] J. P. W. Verbiest, J. M. Weisberg, A. A. Chael, K. J. Lee and D. R. Lorimer, On Pulsar Distance Measurements and Their Uncertainties, ApJ 755 (Aug., 2012) 39, [1206. 0428 ].

[33] R. P. Mignani, G. G. Pavlov and O. Kargaltsev, Optical-Ultraviolet Spectrum and Proper Motion of the Middle-aged Pulsar B1055-52, ApJ 720 (Sept., 2010) 1635-1643, [1007.2940].

[34] O. Adriani, G. C. Barbarino, G. A. Bazilevskaya, R. Bellotti, M. Boezio, E. A. Bogomolov et al., Cosmic-Ray Electron Flux Measured by the PAMELA Experiment between 1 and $625 \mathrm{GeV}$, Physical Review Letters 106 (May, 2011) 201101, [1103.2880]. 
[35] M. Aguilar, D. Aisa, A. Alvino, G. Ambrosi, K. Andeen, L. Arruda et al., Electron and Positron Fluxes in Primary Cosmic Rays Measured with the Alpha Magnetic Spectrometer on the International Space Station, Physical Review Letters 113 (Sept., 2014) 121102.

[36] L. Accardo, M. Aguilar, D. Aisa, A. Alvino, G. Ambrosi, K. Andeen et al., High Statistics Measurement of the Positron Fraction in Primary Cosmic Rays of 0.5-500 GeV with the Alpha Magnetic Spectrometer on the International Space Station, Physical Review Letters 113 (Sept., 2014) 121101.

[37] V. V. Usov, High-frequency emission of X-ray pulsar 1E 2259+586, ApJ 410 (June, 1993) 761-763.

[38] J. Liebert, P. Bergeron and J. B. Holberg, The True Incidence of Magnetism Among Field White Dwarfs, AJ 125 (Jan., 2003) 348-353, [astro-ph/ 0210319 ].

[39] K. Kashiyama, K. Ioka and N. Kawanaka, White dwarf pulsars as possible cosmic ray electron-positron factories, PRD 83 (Jan., 2011) 023002, [1 009.1141$].$

[40] A. A. Abdo, M. Ackermann, M. Ajello, W. B. Atwood, L. Baldini, J. Ballet et al., Gamma-Ray Emission Concurrent with the Nova in the Symbiotic Binary V407 Cygni, Science 329 (Aug., 2010) 817-821, [1008.3912].

[41] S. Razzaque, P. Jean and O. Mena, High energy neutrinos from novae in symbiotic binaries: The case of V407 Cygni, PRD 82 (Dec., 2010) 123012, [1008.5193].

[42] B. D. Metzger, D. Caprioli, I. Vurm, A. M. Beloborodov, I. Bartos and A. Vlasov, Novae as Tevatrons: prospects for CTA and IceCube, MNRAS 457 (Apr., 2016) 1786-1795, [1510 . 07639 ].

[43] I. Büsching, O. C. de Jager, M. S. Potgieter and C. Venter, A Cosmic-Ray Positron Anisotropy due to Two Middle-Aged, Nearby Pulsars?, ApJL 678 (May, 2008) L39, [0 804 . 022 0].

[44] D. Gaggero, L. Maccione, D. Grasso, G. Di Bernardo and C. Evoli, PAMELA and AMS-02 $e^{+}$and $e^{-}$ spectra are reproduced by three-dimensional cosmic-ray modeling, PRD 89 (Apr., 2014) 083007 , [1311.5575]. 